\title{
IDENTIFICATION OF LINES IN THE SPECTRA OF B STARS
}

\author{
By P. SWINGS AND M. NICOLET
}

\begin{abstract}
Identifications of lines in the spectra of B stars are given. About fifty lines for which no previous identifications had been made are interpreted.
\end{abstract}

Within recent years three important lists of absorption lines in B stars have been published ${ }^{\mathrm{I}}$ by $\mathrm{O}$. Struve; ${ }^{2}$ by $\mathrm{O}$. Struve and T. Dunham, Jr.; ${ }^{3}$ and by Roy K. Marshall. ${ }^{4}$ The first list.deals with spectra extending from $\lambda_{3850}$ to $\lambda_{4923 ;}$ the ten stars investigated are of spectral types $\mathrm{O}_{9}, \mathrm{~B} \circ, \mathrm{B}_{1}, \mathrm{~B}_{2}, \mathrm{~B}_{3}, \mathrm{~B}_{5}, \mathrm{~B} 8$. The plates were taken either with the Coudé spectrograph of the Mount Wilson Observatory or with the Bruce spectrograph of the Yerkes Observatory, on Eastman Process emulsion, which gives excellent contrast. Of the total number of lines measured, 73 remained unidentified. The list by O. Struve and T. Dunham, Jr., deals only with the spectrum of the Bo star $23 \tau$ Scorpii. The spectrogram was obtained with the Coudé spectrograph and the roo-inch Mount Wilson reflec- tor, on Eastman Process emulsion, and extends from about $\lambda 3945$ to $\lambda$ 47I 3 . Several new identifications for $C$ III, $N$ III, and $O$ III were added to the first list of Struve, while 53 lines remained unidentified. The study of class B stellar spectra made by $R$. K. Marshall is based on spectra taken with the single-prism spectrograph attached to the $37 \frac{1}{2}$-inch reflector of the University of Michigan. Owing to the unusually high transmission of that optical system, Marshall's list begins at $\lambda$ 3587. I9 A. Most of the plates were taken on Eastman Process emulsion. Of the 534 lines tabulated, almost exactly one-half have not been identified.

× The following abbreviations will be used: O. S. (O. Struve), S. D. (Struve-Dunham) and M (R. K. Marshall).

${ }^{2}$ Ap. J., 74, 225, I931. 3 Ibid., 77, 32 I, I933.

${ }_{4}$ Pub. of the Observatory of the University of Michigan, 5, No. I2, I37, I934.

${ }_{5}$ The region $\lambda \lambda 3850-3950$ is given only for $\gamma$ Pegasi (B2); for wave-lengths longer than 4700 , only the stronger lines could be measured. 
Marshall's list of ultra-violet lines is certainly excellent, but in the ordinary photographic region it is inferior to those of Struve and of Struve and Dunham.

In the following paper we give a few additional identifications. Attention should be drawn toward the following points:

I. In each multiplet the relative intensities of the stellar lines must be compatible with the laboratory intensities.

2. The absolute laboratory intensities have to be considered.

3. The spectral type corresponding to maximum intensity of the stellar line and the behavior of intensity as a function of spectral type must be reasonable.

4. The gradient effect makes all weak absorption lines in dwarfs of type $\mathrm{B}$ relatively stronger than the laboratory intensities might indicate.

5. The atomic weight of the elements and their normal abundances have to be considered.

6. It may happen that for two different spectral types-for example, Bo and B8-lines of the same measured wave-length may have different origins.

7. The difference $\lambda$ obs. $-\lambda$ lab., which is allowed for an identification, depends upon the dispersion in that region and upon the structure of the line (intensity, sharpness).

8. It may happen that a faint line has been omitted in the measures of one particular star.

In this paper none of the lines recently identified by $\mathrm{R}$. K. Marshall $(O$ III $),{ }^{6}$ O. Struve $(C$ III $),{ }^{7}$ J. E. Mack, P. Swings, and $O$. Struve $(C$ IV $),{ }^{8}$ O. Struve and H. Pillans $(S i$ Iv $),{ }^{9}$ D. H. Menzel and R. K. Marshall $(N e \mathrm{II}) ;^{\text {I0 }}$ and E. Mac Cormack $(N e \mathrm{I})^{\mathrm{II}}$ has been listed. When a line of one of the three lists considered here has been identified in one of the other lists, it has been omitted here. When components of a multiplet have been identified by O. S., S. D., or $\mathrm{M}$, the identification of other lines in the same multiplet is comparatively easy; only the new identifications are then given, the symbol of the multiplet being written in brackets.

\footnotetext{
${ }^{6}$ Ap. J., 76, 317, I932.

9 Observatory, 57, I33, I934.

7 Included in list S. D.

ı Proc. Nat. Acad., 19, 879, I933.

${ }^{8}$ Ap. J., 75, 77, I932.

II Pub. A.S.P., 46, 64, I934.
} 
The authors believe that an investigation of the ultra-violet region of $\lambda<3600 \mathrm{~A}$ (by using an aluminized telescope and a quartz or U.V spectrograph) would be fruitful.

TABLE I

$C$ II

\begin{tabular}{|c|c|c|c|c|c|c|}
\hline $\begin{array}{c}\text { Multiplet } \\
\text { Designation }\end{array}$ & $\begin{array}{l}\text { Laboratory } \\
\text { Wave- } \\
\text { Length }\end{array}$ & $\begin{array}{c}\text { Laboratory } \\
\text { Intensity }\end{array}$ & $\begin{array}{l}\text { Stellar } \\
\text { Wave- } \\
\text { Length }\end{array}$ & Author & $\begin{array}{l}\text { Spectral } \\
\text { Types }\end{array}$ & $\begin{array}{l}\text { Identifi- } \\
\text { cation }\end{array}$ \\
\hline $2 \mathrm{p}^{\prime \prime 2} \mathrm{P}-3 \mathrm{p}^{\prime 2} \mathrm{~S}$ & $\begin{array}{l}3868.84 \\
3871.62\end{array}$ & $\begin{array}{l}2 \\
\mathrm{I}\end{array}$ & $\begin{array}{l}8.8 \mathrm{I} \\
\text { I. } 89\end{array}$ & $\begin{array}{l}\mathrm{M} \\
\mathrm{M}\end{array}$ & $\mathrm{B}_{2}$ & $\begin{array}{l}\text { XXX } \\
\text { Bl. He I }\end{array}$ \\
\hline$\left({ }_{3} d^{\prime} 4 F-{ }_{4} f^{4} G\right)$ & $\begin{array}{r}3878.22 \\
79.60 \\
80.59\end{array}$ & $\left.\begin{array}{l}\text { I } \\
I \\
I\end{array}\right\}$ & $\begin{array}{l}8.10 \\
0.38\end{array}$ & $\begin{array}{l}M \\
M\end{array}$ & $\begin{array}{l}\mathrm{B}_{2} \\
\mathrm{BI}_{2},\end{array}$ & $\begin{array}{l}\text { Bl. He I } \\
\text { XXX? }\end{array}$ \\
\hline$\left(3 \mathrm{~d}^{\prime} 4 \mathrm{D}-{ }_{4} \mathrm{f}^{\prime} 4 \mathrm{~F}\right) \ldots$ & $4076 . \infty$ & 7 & $5 \cdot 88$ & O.S. & Bo, 1,2 & Bl. O II \\
\hline $3 \mathrm{~d}^{\prime 2} \mathrm{D}-{ }_{4} \mathrm{f}^{\prime 2} \mathrm{D}$ & $\begin{array}{r}4285.96 \\
96.11\end{array}$ & $\begin{array}{l}\mathrm{I} \\
\mathrm{I}\end{array}$ & $\begin{array}{l}6.24 \\
6.54\end{array}$ & $\begin{array}{l}\text { O.S. } \\
\text { O.S. }\end{array}$ & $\begin{array}{l}\text { B2 } \\
\text { Bo, } 2\end{array}$ & $\begin{array}{l}\mathrm{XXX} \\
\mathrm{XXX}\end{array}$ \\
\hline $3 \mathrm{p}^{\prime} 4 \mathrm{P}-4 \mathrm{~s}^{\prime} 4 \mathrm{P}$. & $\begin{array}{r}4313.50 \\
17.42 \\
18.92 \\
25.88\end{array}$ & $\begin{array}{l}2 \\
4 \\
2 \\
2\end{array}$ & $\begin{array}{l}3.32 \\
7.21 \\
8.45 \\
5.64\end{array}$ & $\begin{array}{l}\text { O.S. } \\
\text { O.S. } \\
\text { O.S. } \\
\text { O.S. }\end{array}$ & $\begin{array}{l}\text { Bo, I, } 2 \\
\text { Bo, I, } 2 \\
\text { Bo, } 2 \\
\text { Bo, I, } 2\end{array}$ & $\begin{array}{l}\text { Bl. O II } \\
\text { Bl. } S \text { II } \\
\text { Bl. O II } \\
\text { Bl. } O \text { II }\end{array}$ \\
\hline Absent........... & $4630 \cdot 5^{2}$ & I & 0.55 & O.S. & Bo, I, 2 & Bl. $O$ II \\
\hline
\end{tabular}

Less probable identifications $(\Delta \lambda$ seems too large):

\begin{tabular}{|c|c|c|c|c|c|c|}
\hline No designation. & $\left\{\begin{array}{l}4292 \cdot 00 \\
4376 \cdot 78 \\
4625 \cdot 7 \mathrm{I}\end{array}\right.$ & $\begin{array}{l}\text { I } \\
\text { I } \\
\text { I }\end{array}$ & $\begin{array}{l}2.55 \\
6.23 \\
5.16\end{array}$ & $\begin{array}{l}\text { S.D. } \\
\text { M } \\
\text { O.S. }\end{array}$ & $\begin{array}{l}\text { Bo } \\
\text { Bo } \\
\text { BI, } 2\end{array}$ & $\begin{array}{l}\text { Bl. } O \text { II } \\
\text { Bl. } O \text { III } \\
\text { XXX }\end{array}$ \\
\hline
\end{tabular}

TABLE II

$N$ II

\begin{tabular}{|c|c|c|c|c|c|c|}
\hline $\begin{array}{c}\text { Multiplet } \\
\text { Designation }\end{array}$ & $\begin{array}{c}\text { Laboratory } \\
\text { Wave- } \\
\text { Length }\end{array}$ & $\begin{array}{l}\text { Laboratory } \\
\text { Intensity }\end{array}$ & $\begin{array}{l}\text { Stellar } \\
\text { Wave- } \\
\text { Length }\end{array}$ & Author & $\begin{array}{l}\text { Spectral } \\
\text { Types }\end{array}$ & $\begin{array}{l}\text { Identifi- } \\
\text { cation }\end{array}$ \\
\hline$\left(3^{3} d^{3} D-{ }_{4} f^{3} D\right)$ & $\begin{array}{r}4 \mathrm{I} 60.8 \\
73.5 \mathrm{I} \\
73.75\end{array}$ & $\begin{array}{l}0 \\
0 \\
0\end{array}$ & $\left.\begin{array}{l}0.62 \\
3.59 \\
4.12 \\
4.06\end{array}\right\}$ & $\begin{array}{l}\text { M } \\
\text { O.S. } \\
\text { M }\end{array}$ & $\begin{array}{l}\mathrm{B} 1,3 \\
\mathrm{~B}_{2}, 3 \\
\mathrm{~B}_{2}, 3\end{array}$ & $\begin{array}{l}\text { XXX } \\
\text { Bl. } S \text { II } \\
\text { Bl. } S \text { II }\end{array}$ \\
\hline $3^{d^{\mathrm{I}} \mathrm{D}}-4^{\mathrm{f}} \mathrm{D} \ldots \ldots$ & $4044 \cdot 75$ & I & $5 . \infty$ & O.S. & $\mathrm{B}_{2}$ & $\mathrm{XXX}$ \\
\hline
\end{tabular}


$C I I, N I I, N I I I$, and $O I I$.- The identifications are given in Tables I, II, III, and IV. ${ }^{12}$

\section{TABLE III}

$N$ III

\begin{tabular}{c|c|c|c|c|c|c}
\hline \hline $\begin{array}{c}\text { Multiplet } \\
\text { Designation }\end{array}$ & $\begin{array}{c}\text { Laboratory } \\
\text { Wave- } \\
\text { Length }\end{array}$ & $\begin{array}{c}\text { Laboratory } \\
\text { Intensity }\end{array}$ & $\begin{array}{c}\text { Stellar } \\
\text { Wave- } \\
\text { Length }\end{array}$ & Author & $\begin{array}{c}\text { Spectral } \\
\text { Types }\end{array}$ & $\begin{array}{c}\text { Identifica- } \\
\text { tion }\end{array}$ \\
\hline $\begin{array}{c}\left(3 \mathrm{~s}^{\prime 2} \mathrm{P}-3 \mathrm{p}^{\prime 2} \mathrm{D}\right) \ldots \\
4 \mathrm{p}^{2} \mathrm{P}-5 \mathrm{~s}^{2} \mathrm{~S} \ldots \ldots\end{array}$ & $\begin{array}{c}42 \mathrm{I} .69 \\
4544.80\end{array}$ & 3 & $\begin{array}{l}5 \cdot 79 \\
4.90\end{array}$ & $\begin{array}{c}\text { S.D. } \\
\text { S.D. }\end{array}$ & $\begin{array}{c}\text { Bo } \\
\text { Bo }\end{array}$ & $\begin{array}{l}\text { Bl. [N II] }] \\
\mathrm{XXX}\end{array}$ \\
\hline
\end{tabular}

Less probable identifications ( $\Delta \lambda$ seems too large)

\begin{tabular}{r|r|r|r|l|l|l}
\hline $3 \mathrm{p}^{\prime 2} \mathrm{D}-3 \mathrm{~d}^{\prime 2} \mathrm{D} \ldots$ & $3934.4 \mathrm{I}$ & 3 & 3.62 & $\mathrm{M}$ & Bo & Bl. Ca II, $S$ II \\
& 38.52 & 4 & $9 . \mathrm{I} 2$ & $\mathrm{M}$ & Bo & XXX? \\
& 42.78 & $\mathrm{I}$ & .3 .46 & $\mathrm{M}$ & Bo & XXX? \\
\hline
\end{tabular}

TABLE IV

$O$ II

\begin{tabular}{|c|c|c|c|c|c|c|}
\hline $\begin{array}{c}\text { Multiplet } \\
\text { Designation }\end{array}$ & $\begin{array}{c}\text { Laboratory } \\
\text { Wave- } \\
\text { Length }\end{array}$ & $\begin{array}{l}\text { Laboratory } \\
\text { Intensity }\end{array}$ & $\begin{array}{l}\text { Stellar } \\
\text { Wave- } \\
\text { Length }\end{array}$ & Author & $\begin{array}{c}\text { Spectral } \\
\text { Types }\end{array}$ & $\begin{array}{l}\text { Identifica- } \\
\text { tion }\end{array}$ \\
\hline $4 \mathrm{p}^{2} \mathrm{P}-{\overline{4 \mathrm{~d}^{2}}}^{2} \mathrm{D} \ldots$ & 3785.01 & o & $5 \cdot 36$ & $\mathbf{M}$ & $\mathrm{BI}, 2$ & $\mathrm{XXX}$ \\
\hline $4 \mathrm{~s}^{2} \mathrm{P}-\overline{4 \mathrm{p}}^{2} \mathrm{D} \ldots$ & $3838.4 \mathrm{I}$ & $\circ$ & 8.30 & M & $\mathrm{BI}, 2$ & Bl. $H e$ I, $N$ II \\
\hline$\left(3^{4} \mathrm{~d} F-4^{4} \mathrm{~F}\right) \ldots$ & $\begin{array}{r}4026.40 \\
33.18 \\
44.96 \\
46.15\end{array}$ & $\begin{array}{l}0 \\
0 \\
0 \\
0\end{array}$ & $\begin{array}{l}6.3 I \\
2.90 \\
5.00 \\
5.80\end{array}$ & $\begin{array}{l}\text { S.D. } \\
\text { O.S. } \\
\text { O.S. } \\
\text { M }\end{array}$ & $\begin{array}{l}\mathrm{Bo} \\
\mathrm{B} 2 \\
\mathrm{~B} 2 \\
\mathrm{Bo}\end{array}$ & $\begin{array}{l}\text { Bl. } H e \mathrm{I}, N \text { II } \\
\text { XXX } \\
\text { XXX } \\
\mathrm{XXX}\end{array}$ \\
\hline $\mathrm{e}^{4} \mathrm{P}^{0}-z^{4} \mathrm{D} \ldots$ & $4 \mathrm{IO} 3.0 \mathrm{I}$ & 5 & 3.40 & $\begin{array}{l}\text { O.S. and } \\
M\end{array}$ & $\mathrm{O}_{9}, \mathrm{Bo}, 2$ & Bl. $N$ III \\
\hline 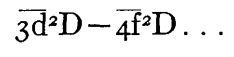 & 4342.83 & I & 2.90 & O.S. & Bo, I & Bl. $O$ II \\
\hline$\left(3^{2} \mathrm{~d}-4^{4} \mathrm{D}\right) \ldots$ & 4482.85 & $\circ$ & 2.98 & S.D. & Bo & Bl. $O$ III \\
\hline$\left(3^{2} \mathrm{~d}-4 \mathrm{f}^{2} \mathrm{D}\right) \ldots$ & $\begin{array}{r}46 \mathrm{r} 3.67 \\
21.28\end{array}$ & $\begin{array}{l}\text { I } \\
\text { O }\end{array}$ & $\begin{array}{l}3.84 \\
\text { I. } 49\end{array}$ & $\begin{array}{l}\text { S.D. } \\
\text { S.D. }\end{array}$ & $\begin{array}{l}\text { Bo } \\
\text { Bo }\end{array}$ & $\begin{array}{l}\text { Bl. } N \text { II } \\
\text { Bl. } N \text { II }\end{array}$ \\
\hline$\left(3 \mathrm{~d}^{2} \mathrm{D}-4^{2} \mathrm{f}\right) \ldots$ & 4707.80 & $\circ$ & $7 \cdot 98$ & O.S. & Bo, I, 2 & $\mathrm{XXX}$ \\
\hline
\end{tabular}

I2 In these tables the symbol xxx means that no identification existed previously for the line; in case of blending, the blending line is indicated. 
Ne II.-A few remarks may be made concerning the identification of $N e$ II lines by D. H. Menzel and R. K. Marshall. ${ }^{10}$

I. In $3 \mathrm{~s}^{4} \mathrm{P}-3 \mathrm{p}^{4} \mathrm{P}^{0}$ it does not seem possible that the strong $N e$ II line $\lambda 3766.29$ which is at a distance of $4 A$ from $H \iota$ could have been masked by $H \iota$.

2. In $3 \mathrm{P}^{2} \mathrm{P}^{0}-3 \mathrm{~d}^{2} \mathrm{D}$ the identifications are rather doubtful, as the strongest line $\lambda 3829.77$ does not appear in ro Lacertae (an O9 spectrum with very numerous and sharp lines), although the region from $\lambda_{3} 820$ to $\lambda_{3} 835$ in ro Lacertae is completely free from lines.

3. In $3 \mathrm{~d}^{4} \mathrm{D}-4^{4} \mathrm{D}^{\circ}$ three lines by O. S. and S. D. are identified by Menzel and Marshall; it is also possible to identify $\lambda_{4217.22}$ ( $\tau$ Scorpii, Bo, int. 2 ) with $\lambda$ lab. 42 I 7.I 5 (int. 3 ).

4. In $3 \mathrm{~d}^{4} \mathrm{~F}-4^{\mathrm{f}}{ }^{4} \mathrm{G}^{0}$ two identifications may be added to the two made previously by Menzel-Marshall:

\begin{tabular}{|c|c|c|c|c|c|c|}
\hline $\begin{array}{c}\text { Multiplet } \\
\text { Designation }\end{array}$ & $\begin{array}{c}\text { Laboratory } \\
\text { Wave- } \\
\text { Length }\end{array}$ & $\begin{array}{l}\text { Laboratory } \\
\text { Intensity }\end{array}$ & $\begin{array}{l}\text { Stellar } \\
\text { Wave- } \\
\text { Length }\end{array}$ & Author & $\begin{array}{l}\text { Spectral } \\
\text { Types }\end{array}$ & $\begin{array}{l}\text { Identifica- } \\
\text { tion }\end{array}$ \\
\hline \multirow[t]{2}{*}{$\left(3^{d^{4}} \mathrm{~F}-4^{\mathrm{f}^{4}} \mathrm{G}^{0}\right) \ldots$} & & 6 & $\left\{\begin{array}{l}0.53 \\
0.47\end{array}\right.$ & $\begin{array}{l}\text { S.D. } \\
\text { M }\end{array}$ & $\begin{array}{l}\text { Bo } \\
\text { Bo }\end{array}$ & Bl. $N$ III \\
\hline & $4428 \cdot 54$ & 6 & $\left\{\begin{array}{l}8.53 \\
8.44\end{array}\right.$ & $\begin{array}{l}\text { S.D. } \\
\text { M }\end{array}$ & $\begin{array}{l}\text { Bo? } \\
\text { Bo\} }\end{array}$ & Bl. [ $N$ II] \\
\hline
\end{tabular}

5. In $3 \mathrm{~d}^{4} \mathrm{~F}-{ }_{4} \mathrm{f}^{4} \mathrm{~F}^{0}$ other identifications in $\tau$ Scorpii (S.D.) are allowed:

\begin{tabular}{l|r|l|l|l|l|l}
\hline \hline$\left(3 \mathrm{~d} 4 \mathrm{~F}-4^{\mathrm{f}} \mathrm{F}^{\circ}\right) \ldots$ & 4369.77 & 5 & 9.33 & S.D. & Bo & Bl. $O$ II \\
& 4397.94 & 6 & 8.01 & S.D. & Bo & XXX \\
& 4430.90 & 4 & 0.99 & S.D. & Bo & Bl. [S II] \\
42.67 & 3 & 2.95 & S.D. & Bo & Bl. O II \\
& 46.46 & 3 & 6.97 & S.D. & Bo & Bl. $N$ II, $O$ II \\
\hline
\end{tabular}

6. An identification may be made in $3 \mathrm{~d}^{2} \mathrm{P}-4 \mathrm{f}^{2} \mathrm{D}^{0}$ :

\begin{tabular}{|c|c|c|c|c|c|c|}
\hline $3 \mathrm{~d}^{2} \mathrm{P}-4 \mathrm{f}^{2} \mathrm{D}^{0} \ldots$ & $\begin{array}{r}45 \text { I I . } 29 \\
\text { II } 37\end{array}$ & $\begin{array}{l}2\} \\
4\}\end{array}$ & $\begin{array}{l}\text { II. I3 } \\
\text { Io. } 88\end{array}$ & $\begin{array}{l}\text { M } \\
\text { S.D. and } \\
\text { O.S. }\end{array}$ & $\left.\begin{array}{c}\text { Bo } \\
\mathrm{O}_{9}, \mathrm{Bo}\end{array}\right\}$ & Bl. $N$ III \\
\hline
\end{tabular}


$N a I I .{ }^{\text {r3 }-T h e ~} N a$ II absorption lines having a wave-length greater than 3580 A require an excitation potential of more than 32 volts; thus they should be strong only in the hottest stars. $\lambda_{363}{ }_{3} .37$ (int. 8) of $N a$ II may tentatively account for $\lambda_{3} 6_{3}{ }_{1} 6_{7}$ (class $\mathrm{O}_{9}$ ). An examination of the ultra-violet parts of a few $\mathrm{O}_{5}-\mathrm{O} 8$ spectra would be interesting.

Na III and Mg III.-The lower excitation potentials of the visible lines are:

$$
\begin{array}{ll}
\text { For } N a \text { III } & : \geq 57 \text { volts }, \\
\text { For } M g \text { III } & : \geq 65 \text { volts }{ }^{14}
\end{array}
$$

Thus it seems impossible to expect these lines in B-type spectra.

$A l I I$ and III.-Marshall casts suspicion upon the identification of $A l \mathrm{II}$ and $A l$ III. The identification of $A l \mathrm{III}$ is absolutely safe and some of the lines are actually strong (e.g., $\lambda \lambda 4^{1} 5^{\circ}$, 4480, 45 I2.5, and 4529), as may be seen on the illustrations of the spectrum of $\gamma$ Pegasi given in Struve's complete paper. ${ }^{15}$

Besides $\lambda \lambda_{3601.73}$ (int. 4) and 36r 2.26 (int. 2) observed by Marshall in a Pegasi and $\beta$ Canis Majoris and which are certainly due to $A l \mathrm{III}$, two lines in the visible part of Marshall's list, $\lambda \lambda{ }_{4} \mathrm{I} 50.05$ and 4529.45, have their origin in $A l$ III.

$A l$ II seems also to be definitely present. Several lines in Marshall's list may be attributed to $A l$ II by comparison with Struve's complete list. In addition to those lines a few more identifications are indicated in Table V.

Table $\mathrm{V}$ shows that $A l \mathrm{II}$ is certainly present in B stars. It may be recalled that in the Sun the abundances of $A l$ and $S i$ are of the same order $\left(\mu_{A l} \sim\right.$ IO $\left.^{-1} \mu_{S i}\right)$. The three first ionization potentials are similar:

$$
\begin{array}{lll}
A l \text { I } 5.96 ; & A l \text { II } 18.73 ; & A l \text { III } 28.3 \text { I } \\
S i \text { I } 8.12 ; & S i \text { II } 16.27 ; & S i \text { III } 33.30 .
\end{array}
$$

$P I I I$ and $I V$.- For $P$ III the identifications are indicated in Table VI.

${ }^{13}$ Questioned by Marshall, op. cit., p. I53.

${ }^{14}$ Kindly communicated by Dr. B. Edlén and Dr. J. Söderqvist.

${ }^{15}$ Op. cit., p. 248, Pl. XI. 
TABLE V

$A l$ II

\begin{tabular}{|c|c|c|c|c|c|c|}
\hline $\begin{array}{c}\text { Multiplet } \\
\text { Designation }\end{array}$ & $\begin{array}{c}\text { Laboratory } \\
\text { Wave- } \\
\text { Length }\end{array}$ & $\begin{array}{l}\text { Laboratory } \\
\text { Intensity }\end{array}$ & $\begin{array}{l}\text { Stellar } \\
\text { Wave- } \\
\text { Length }\end{array}$ & Author & $\begin{array}{l}\text { Spectral } \\
\text { Types }\end{array}$ & Identification \\
\hline$\left(4^{3} \mathrm{P}^{0}-5^{3} \mathrm{D}\right)$ & $\begin{array}{r}3649.20 \\
5 \mathrm{I} .06\end{array}$ & $\begin{array}{l}\mathrm{I} \cdot 5 \\
6\end{array}$ & $\begin{array}{l}9.58 \\
\text { I. } 14\end{array}$ & $\begin{array}{l}M \\
M\end{array}$ & $\begin{array}{l}\mathrm{B}_{2} \\
\mathrm{~B}_{2}\end{array}$ & $\underset{X X X}{X X X}$ \\
\hline $4^{\mathrm{x}} \mathrm{P}^{0}-6^{\mathrm{I}} \mathrm{D} \ldots$ & 3703.22 & 4 & $3 \cdot 38$ & M & $\mathrm{B}_{2}$ & $\mathrm{XXX}$ \\
\hline $4^{3} \mathrm{D}-\mathrm{I} 2^{3} \mathrm{~F} \ldots$ & $3734 \cdot 7$ & I & $4 \cdot 33$ & $\mathbf{M}$ & $\ldots$ & Bl. $H_{\lambda}$ \\
\hline $4^{3} \mathrm{P}-6^{3} \mathrm{~S}$ & $\begin{array}{r}373 \mathrm{I} .95 \\
3.9 \mathrm{I} \\
8.00\end{array}$ & $\begin{array}{l}\text { I } \\
2 \\
3\end{array}$ & $\begin{array}{r}2.04 \\
4 \cdot 33 \\
\left\{\begin{array}{l}7.87 \\
7 \cdot 35\end{array}\right.\end{array}$ & $\begin{array}{l}M \\
M \\
L^{*} \\
M\end{array}$ & $\begin{array}{l}\mathrm{B}_{2,3}, \\
\ldots \ldots \\
\mathrm{B}_{3} \\
\mathrm{~B}_{5}\end{array}$ & $\begin{array}{l}\mathrm{XXX} \\
\mathrm{Bl} . H_{\lambda} \\
\mathrm{XXX} \\
\mathrm{XXX}\end{array}$ \\
\hline $5^{\mathrm{I}} \mathrm{S}-\mathrm{I} 2^{\mathrm{I}} \mathrm{P}$. & $3753 \cdot 10$ & I & $3 \cdot 44$ & 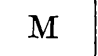 & $\mathrm{B}_{2}, 8$ & $\mathrm{XXX}$ \\
\hline $5^{3} \mathrm{~S}-8{ }^{3} \mathrm{P} \ldots$ & $3774 \cdot 3$ & $\circ$ & $3 \cdot 73$ & M & $\mathrm{B}_{2,}, 3,5$ & $\mathrm{XXX}$ ? \\
\hline $4^{3} D-I^{3} F \ldots$ & 3842.2 & 3 & 2.74 & M & $\mathrm{B}_{5}$ & Bl. $O$ II, $N$ II? \\
\hline$\left(4^{3} \mathrm{D}-\mathrm{ro}^{3} \mathrm{~F}^{0}\right)$ & $\begin{array}{r}3996.08 \\
.16 \\
.32 \\
.38\end{array}$ & $\left.\begin{array}{l}\text { I } \\
4 \\
0.5 \\
3\end{array}\right\}$ & 6.66 & $M$ & $\mathrm{~B}_{5}$ & $\mathrm{xxx}$ \\
\hline $5^{\mathrm{r} S}-\mathrm{r} \mathrm{o}^{\mathrm{T}} \mathrm{P} \ldots \ldots$ & $4009 \cdot 58$ & I & $9 \cdot 32$ & M & $\mathrm{B}_{2,3}, 5,8$ & Bl. $H e \mathrm{I}$ \\
\hline
\end{tabular}

* Measured by H. M. Losh, in $\zeta$ Tauri (Pub. Obs. U. of Michigan, 4, I, Table ro, 193r).

TABLE VI

$P$ III

\begin{tabular}{|c|c|c|c|c|c|c|}
\hline $\begin{array}{c}\text { Multiplet } \\
\text { Designation }\end{array}$ & $\begin{array}{c}\text { Laboratory } \\
\text { Wave- } \\
\text { Length }\end{array}$ & $\begin{array}{c}\text { Laboratory } \\
\text { Intensity }\end{array}$ & $\begin{array}{l}\text { Stellar } \\
\text { Wave- } \\
\text { Length }\end{array}$ & Author & $\begin{array}{l}\text { Spectral } \\
\text { Types }\end{array}$ & Identification \\
\hline $4 \mathrm{~S}^{4} \mathrm{P}^{0}-4 \mathrm{P}^{4} \mathrm{P}$ & $\begin{array}{c}3895.03 \\
3904 \cdot 79 \\
22.72 \\
33.38 \\
5 \mathrm{I} \cdot 5 \mathrm{I} * \\
57.64 \\
97.17\end{array}$ & $\begin{array}{l}6 \\
6 \\
4 \\
4 \\
5 \\
6 \\
5\end{array}$ & $\begin{array}{l}5.56 \\
4.88 \\
2.79 \\
3.62 \\
2.75 \\
7.54 \\
6.66\end{array}$ & $\begin{array}{l}\mathbf{M} \\
\mathbf{M} \\
\mathbf{M} \\
\mathbf{M} \\
\mathbf{M} \\
\mathbf{M} \\
\mathrm{M}\end{array}$ & $\begin{array}{l}\text { B I, 2 } \\
\text { Bo, I } \\
\text { Bo, I } \\
\text { Bo, I } \\
\text { BI } \\
\text { Bo; I, } 2 \\
\text { Bo, I }\end{array}$ & $\begin{array}{l}\mathrm{XXX} \\
\mathrm{XXX} \\
\mathrm{XXX} \\
\text { Bl. Ca II, } S \text { II } \\
\text { XXX } \\
\mathrm{XXX} \\
\mathrm{XXX}\end{array}$ \\
\hline
\end{tabular}

* This line is quoted by Marshall as being of poor quality; the wave-length was not measured in the Br star $\beta$ Cephei, but in the B 5 p star 67 Ophiuchi; the lines in $\beta$ Cephei and in 67 Ophiuchi have probably different origin. 
$P$ behaves similarly to $S i$, as is shown by the ionization potentials:

$P$ I II.II $; \quad P$ II 19.8 I $; \quad P$ III $30.23 ; \quad P$ IV 5I.I

$S i$ I 8. I2 $; S i$ II I6.27; $S i$ III 33.30; $S i$ IV 44.9I .

Bowen $^{\text {I6 }}$ has found a line of $P$ IV at $\lambda 4_{4249.57}$ (int. 6) having the designation $4^{\mathrm{T}} \mathrm{S}-4^{\mathrm{x}} \mathrm{P}^{\circ}$ (lower E.P.-28.9 volts), which could possibly

TABLE VII

$S$ II

\begin{tabular}{|c|c|c|c|c|c|c|}
\hline $\begin{array}{l}\text { Multiplet } \\
\text { Designation }\end{array}$ & $\begin{array}{c}\text { Laboratory } \\
\text { Wave- } \\
\text { Length }\end{array}$ & $\begin{array}{c}\text { Laboratory } \\
\text { Intensity }\end{array}$ & $\begin{array}{l}\text { Stellar } \\
\text { Wave- } \\
\text { Length }\end{array}$ & Author & $\begin{array}{c}\text { Spectral } \\
\text { Types }\end{array}$ & Identification \\
\hline $4 \mathrm{p}^{\prime 2} \mathrm{D}^{0}-4 \mathrm{~s}^{2} \mathrm{P}$. & 3669.03 & 4 & 9.23 & M & $\mathrm{B}_{2}$ & $\mathrm{XXX}$ \\
\hline $4 \mathrm{P}^{2} \mathrm{~S}^{0}-{ }^{2} \mathrm{~s}^{2} \mathrm{P} \ldots$ & $3783 \cdot 13$ & 3 & 2.78 & M & $\mathrm{B}_{2}$ & $\mathrm{XXX}$ \\
\hline $4 \mathrm{P}^{4} \mathrm{P}^{0}-4 \mathrm{~d}^{4} \mathrm{P}$ & $\begin{array}{r}379^{2} .46 \\
3802.68 \\
60.66 \\
9^{2} .3^{2}\end{array}$ & $\begin{array}{l}5 \\
1 \\
3 \\
5\end{array}$ & $\begin{array}{l}2.63 \\
3.12 \\
0.58 \\
2.21\end{array}$ & $\begin{array}{l}\mathbf{M} \\
\mathbf{M} \\
\mathbf{M} \\
\mathbf{M}\end{array}$ & $\begin{array}{l}\mathrm{B} 2,3 \\
\mathrm{~B}_{2} \\
\mathrm{~B} 2,3,5 \\
\mathrm{~B} 2,3,5\end{array}$ & $\begin{array}{l}\text { XXX } \\
\text { Bl. O II } \\
\text { XXX } \\
\text { XXX }\end{array}$ \\
\hline$\left(4 \mathrm{p}^{4} \mathrm{D}^{0}-4 \mathrm{~d}^{4} \mathrm{D}\right) \ldots$ & $\begin{array}{r}3939.5^{2} \\
47.02 \\
50.45 \\
63.14 \\
70.68 \\
90.94 \\
4003.90\end{array}$ & $\begin{array}{l}\circ \\
2 \\
0 \\
3 \\
2 \\
8 \\
3\end{array}$ & $\begin{array}{l}9.12 \\
7.28 \\
9.67 \\
2.64 \\
0.10 \\
0.98 \\
3.58\end{array}$ & $\begin{array}{l}\text { M } \\
M \\
M \\
M \\
M \\
M \\
\text { S.D. }\end{array}$ & $\begin{array}{l}\mathrm{BI}, 2,3 \\
\mathrm{Bo}, \mathrm{I}, 2 \\
\mathrm{Bo}, \mathrm{I}, 2,3 \\
\mathrm{Bo}, 5 \\
\ldots \ldots \ldots \\
\mathrm{BI}, 2,5 \\
\mathrm{Bo}\end{array}$ & $\begin{array}{l}\text { XXX? } \\
\text { XXX? } \\
\text { XXX? } \\
\text { Bl. O II } \\
\text { Bl. H } \epsilon ? \\
\text { XXX } \\
\text { Bl. } N \text { III }\end{array}$ \\
\hline$\left(4 \mathrm{p}^{2} \mathrm{D}-4^{\mathrm{d}^{2} \mathrm{~F}}\right)$. & $4009 \cdot 4^{\mathrm{I}}$ & 2 & 9.28 & o.s. & & Bl. $H e \mathrm{I}[C \mathrm{II}]$ \\
\hline$\left(3 \mathrm{~d}^{2} \mathrm{~F}-4 \mathrm{p}^{\prime 2} \mathrm{~F}^{0}\right) \ldots$ & 3993.49 & 6 & $3.6 \mathrm{I}$ & $\mathrm{M}$ & $\mathrm{B}_{2}$ & $\mathrm{XXX}$ \\
\hline
\end{tabular}

explain the unidentified $\lambda_{425}$. Io (int. I) of 1o Lacertae (class O9) and $\lambda{ }_{4249.89}$ (int. I) of $\tau$ Scorpii (class Bo).

$S I I$ and III.-According to Marshall, ${ }^{17}$ Gilles's tables are untrustworthy, but the investigations of O. Bartelt and L. Eckstein ${ }^{18}$ have brought new material which has allowed the identifications listed in Tables VII and VIII.

I6 Phys. Rev., 39, 8, 1932.

${ }^{7} \mathrm{Op}$. cit., p. I 52 .

${ }^{18}$ Zs. für Phys., 86, 77, 1933; Zs. für Astroph., 7, 272, 1933. 
TABLE VIII

$S$ III

\begin{tabular}{|c|c|c|c|c|c|c|}
\hline $\begin{array}{c}\text { Multiplet } \\
\text { Designation }\end{array}$ & $\begin{array}{c}\text { Laboratory } \\
\text { Wave- } \\
\text { Length }\end{array}$ & $\begin{array}{c}\text { Laboratory } \\
\text { Intensity }\end{array}$ & $\begin{array}{l}\text { Stellar } \\
\text { Wave- } \\
\text { Length }\end{array}$ & Author & $\begin{array}{l}\text { Spectral } \\
\text { Types }\end{array}$ & $\begin{array}{l}\text { Identifica- } \\
\text { tion }\end{array}$ \\
\hline$\left(4 \mathrm{~S}^{3} \mathrm{P}^{0}-4 \mathrm{p}^{3} \mathrm{P}\right)$ & $\begin{array}{r}3837.79 \\
60.64\end{array}$ & $\begin{array}{l}5 \\
4\end{array}$ & $\begin{array}{l}7.33 \\
0.58\end{array}$ & $\begin{array}{l}M \\
M\end{array}$ & $\begin{array}{l}\mathrm{BI}, 2 \\
\mathrm{BI}, 2,3\end{array}$ & $\underset{X X X}{\mathrm{XXX}}$ \\
\hline$\left(3 d^{3} D^{0}-4 p^{3} D\right)$ & $\begin{array}{l}4499.29 \\
45^{27} \cdot 9^{6}\end{array}$ & $\begin{array}{l}\circ \\
0\end{array}$ & $\begin{array}{l}9.40 \\
7.18\end{array}$ & $\begin{array}{l}\text { S.D. } \\
\text { M }\end{array}$ & $\begin{array}{l}\text { Bo } \\
\text { Bo }\end{array}$ & $\begin{array}{l}\text { XXX? } \\
\text { XXX?? }\end{array}$ \\
\hline
\end{tabular}

Ca III.--There are two laboratory lines in the astrophysical region:

\begin{tabular}{l|l|l|l|l|l|l}
\hline $4 \mathrm{~s}_{3}-4 \mathrm{p}_{\mathrm{ro}} \ldots \ldots \ldots \ldots$ & $376 \mathrm{r} .62$ & 6 & $\mathrm{I} .27$ & $\mathrm{M}$ & $\mathrm{O}_{9}$ & $\mathrm{XXX}$ \\
$4 \mathrm{~s}_{2}-4 \mathrm{p}_{\mathrm{ro}}{ }^{*} \ldots \ldots \ldots$ & $408 \mathrm{r} .74$ & 5 & $\mathrm{I} .83$ & $\mathrm{M}$ & $\mathrm{O}_{9}-\mathrm{Bo}$ & $\mathrm{XXX}$ \\
\hline
\end{tabular}

* This second identification is somewhat doubtful, as there exists some incompatibility between the measures of S.D. and $M$ near $\lambda$ 408I A.

Ti II.-Possible identifications are indicated in Table IX.

TABLE IX

\begin{tabular}{|c|c|c|c|c|c|c|}
\hline $\begin{array}{l}\text { Multiplet } \\
\text { Designation }\end{array}$ & $\begin{array}{c}\text { Laboratory } \\
\text { Wave- } \\
\text { Length }\end{array}$ & $\begin{array}{l}\text { Laboratory } \\
\text { Intensity }\end{array}$ & $\begin{array}{l}\text { Stellar } \\
\text { Wave- } \\
\text { - Length }\end{array}$ & Author & $\begin{array}{c}\text { Spectral } \\
\text { Types }\end{array}$ & $\begin{array}{l}\text { Identifica- } \\
\text { tion }\end{array}$ \\
\hline $\mathrm{b}^{2} \mathrm{D}-\mathrm{y}^{2} \mathrm{D}^{0}$ & $\begin{array}{r}3741.68 \\
57.69 \\
76.06\end{array}$ & $\begin{array}{l}(50) \\
(30) \\
(6)\end{array}$ & $\begin{array}{l}\text { I. } 85 \\
7.70 \\
5.97\end{array}$ & $\begin{array}{l}\mathbf{M} \\
\mathbf{M} \\
\mathbf{M}\end{array}$ & $\begin{array}{l}\mathrm{B}_{3} \\
\mathrm{~B} 8 \\
\mathrm{~B}_{5}-\mathrm{B} 8\end{array}$ & $\begin{array}{l}\mathrm{XXX} \\
\mathrm{XXX} \\
\mathrm{XXX}\end{array}$ \\
\hline
\end{tabular}

$A I I$. - When comparing all the multiplets of $A$ II given by $\mathrm{De}$ Bruin $^{19}$ with the absorption lines of classes $\mathrm{B}_{0}, \mathrm{~B}_{\mathrm{I}}$. and $\mathrm{B}_{2}$, we find a percentage of coincidences as large as in the case of $\mathrm{Ne}$ II. The number of coincidences observed is obviously greater than the number of chance coincidences calculated by the Russell-Bowen formula; in addition, the intensities observed agree quite well with the laboratory intensities. If the presence of $A$ II could be verified with certainty, an important number of lines of B stars would be identified $;^{20}$ these lines appear where they ought to (maximum at $\mathrm{B}_{\mathrm{I}}-\mathrm{B}_{2}$ ), owing

${ }_{9}$ Zs. für Phys., 48, 62, I928; 5r, 108, I928; 6r, 307, I930.

${ }^{20}$ More than twelve, which had heretofore no other suggested identification. 
to their ionization and excitation potentials, which are lower than for $\mathrm{Ne}$ II.

Ionization potentials:

$\mathrm{Ne}$ I 2 I .47 volts ; $N e$ II 40.9 volts ;

$A$ I 15.69 volts ; $A$ II 27.72 volts

Excitation potential of lower level:

$N e$ II : between 27 and 28 volts;

$A$ II : between 17 and 22 volts.

Arguments against these identifications are the atomic weight of argon (39.94) and the fact that it has never been observed in stellar or nebular spectra. These arguments do not seem convincing. The discovery of $A$ II in $\mathrm{B}$ stars would be of interest, in connection with a recent paper by $H$. N. Russell and D. H. Menzel. ${ }^{2 \pi}$

The question of $A$ II is being investigated here; the results will appear later.

UNIVERSITY OF LIÉge (BELGTUM)

Department OF Astrophysics May Io, 1934

${ }^{2 x}$ Proc. Nat. Acad., 19, 997, r933. 